MATHEMATICS OF COMPUTATION

Volume 75, Number 253, Pages 167-181

S 0025-5718(05)01793-X

Article electronically published on September 29, 2005

\title{
THE MULTI-SYMPLECTICITY OF PARTITIONED RUNGE-KUTTA METHODS FOR HAMILTONIAN PDES
}

\author{
JIALIN HONG, HONGYU LIU, AND GENG SUN
}

\begin{abstract}
In this article we consider partitioned Runge-Kutta (PRK) methods for Hamiltonian partial differential equations (PDEs) and present some sufficient conditions for multi-symplecticity of PRK methods of Hamiltonian PDEs.
\end{abstract}

\section{INTRODUCTION}

It has been widely recognized that the symplectic integrator has the numerical superiority when applied to solving Hamiltonian ODEs. A systemic theory of symplectic integrators for Hamiltonian ODEs has been established by some authors. The Runge-Kutta methods play an important role in numerically solving differential equations (see [1, [3, [4, [6]-[16] and the references therein). The symplectic condition of Runge-Kutta methods was found independently by Lasagni, Sanz-Serna and Suris in 1988 (see [10, 12, 15] and the references therein). The numerical analysis has been investigated and developed by some authors (see [4, [6], [7, [10][16] and the references therein). Some characterizations of symplectic partitioned Runge-Kutta methods, which are very useful for the construction of symplectic schemes for solving numerical Hamiltonian problems, were obtained by Sanz-Serna in 10, Sun in 13, 14 and Suris in 16 and recently discussed by Marsden and West in 8 . Reich in 9 considered Hamiltonian wave equations and showed that the Gauss-Legendre discretization applied to the scalar wave equation (and Schrödinger equation) in both the time and space directions leads to a multi-symplectic integrator. Motivated by [6, [7], [9], [11, [13, [14, [16, we considered the following questions. Are there any multi-symplectic partitioned Runge-Kutta methods for Hamiltonian PDEs? What is the characterization of multi-symplectic partitioned RungeKutta methods for the general case of Hamiltonian PDEs? The answer to the first question is obviously affirmative. The last question is closely related to the construction of higher-order multi-symplectic schemes for Hamiltonian PDEs. In this

Received by the editor November 23, 2004.

2000 Mathematics Subject Classification. Primary 65P10, 58F05; Secondary 65M06, 65M99, 65N06, 65N99, 58F99, 58G99.

Key words and phrases. Partitioned Runge-Kutta method, multi-symplecticity, Hamiltonian partial differential equation.

The first author was supported by the Director Innovation Foundation of ICMSEC and AMSS, the Foundation of CAS, the NNSFC (No.19971089, No.10371128) and the Special Funds for Major State Basic Research Projects of China G1999032804.

The third author was supported in part by the Director Innovation Foundation of the Institute of Mathematics and the AMSS. 
article we consider the general case of Hamiltonian PDEs, investigate the multisymplecticity of partitioned Runge-Kutta methods, and then present some conditions for multi-symplectic partitioned Runge-Kutta methods. In the rest of this section we introduce some basic concepts on multi-symplectic discretization and multi-symplecticity of Hamiltonian PDEs and give an extension version of Reich's result on the multi-symplecticity of Gauss-Legendre methods for the general case of Hamiltonian PDEs. In section 2 we present conditions for multi-symplecticity of partitioned Runge-Kutta methods when applied to a Hamiltonian PDE. In section 3, the multi-symplecticity of partitioned Runge-Kutta methods for the wave equation is discussed. In section 4 we investigate conservative properties of energy and momentum for Runge-Kutta methods of linear Hamiltonian PDEs. In what follows we assume that all numerical methods proposed are numerically solvable and only focus on the multi-sympleciticity of methods.

Consider the Hamiltonian partial differential equation

$$
M z_{t}+K z_{x}=\nabla_{z} S(z), \quad(x, t) \in \Omega \subset R^{2},
$$

where $M$ and $K$ are skew-symmetric matrices and $S$ is a real smooth function of the variable $z$. As is well known, some very important partial differential equations can be rewritten in this form (see [2, $[9]$ and the references therein). The following is its multi-symplectic conservation law

$$
\frac{\partial \omega(U, V)}{\partial t}+\frac{\partial \kappa(U, V)}{\partial x}=0,
$$

where

$$
\omega(U, V)=U^{T} M^{T} V, \quad \kappa(U, V)=U^{T} K^{T} V,
$$

and $U(x, t)$ and $V(x, t)$ are solutions of the variational equation

$$
M d z_{t}+K d z_{x}=D_{z z} S(z) d z .
$$

In order to study the multi-symplecticity-preserving Runge-Kutta method, we introduce a uniform grid $\left(x_{j}, t_{k}\right) \in R^{2}$ with mesh length $\Delta t$ in the $t$ direction and mesh length $\Delta x$ in the $x$ direction. The value of the function $\psi(x, t)$ at the mesh point $\left(x_{j}, t_{k}\right)$ is denoted by $\psi_{j, k}$. The equations (1.1), (1.2), and (1.3) can be, respectively, schemed numerically as

$$
\begin{gathered}
M \partial_{t}^{j, k} z_{j, k}+K \partial_{x}^{j, k} z_{j, k}=\left(\nabla_{z} S_{j, k}\right)_{j, k} \\
\partial_{t}^{j, k} \omega_{j, k}+\partial_{x}^{j, k} \kappa_{j, k}=0 \\
M \partial_{t}^{j, k}(d z)_{j, k}+K \partial_{x}^{j, k}(d z)_{j, k}=\left(D_{z z}^{j, k} S_{j, k}\right)(d z)_{j, k},
\end{gathered}
$$

where $S_{j, k}=S\left(z_{j, k}, x_{j}, t_{k}\right)$,

$$
\omega_{j, k}=\left\langle M U_{j, k}, V_{j, k}\right\rangle, \quad \kappa_{j, k}=\left\langle K U_{j, k}, V_{j, k}\right\rangle,
$$

$U_{j, k}$ and $V_{j, k}$ are solutions of (1.6), and $\partial_{t}^{j, k}, \partial_{x}^{j, k}$ are discretizations of the derivatives $\partial_{t}$ and $\partial_{x}$, respectively. The following definition is from [2].

Definition 1.1. The numerical scheme (1.4) is called multi-symplectic if (1.5) is a discrete conservation law of (1.4). 
To simplify notation, let the starting point $\left(x_{0}, t_{0}\right)=(0,0)$ in the numerical methods proposed throughout this paper. The Runge-Kutta method for equation (1.1) is

$$
\begin{gathered}
Z_{m k}=z_{m}^{\mathbf{0}}+\Delta t \sum_{j=1}^{r} a_{k j} \partial_{t} Z_{m j}, \\
z_{m}^{\mathbf{1}}=z_{m}^{\mathbf{0}}+\Delta t \sum_{k=1}^{r} b_{k} \partial_{t} Z_{m k}, \\
Z_{m k}=z_{\mathbf{0}}^{k}+\Delta x \sum_{n=1}^{s} \tilde{a}_{m n} \partial_{x} Z_{n k}, \\
z_{\mathbf{1}}^{k}=z_{\mathbf{0}}^{k}+\Delta x \sum_{m=1}^{s} \tilde{b}_{m} \partial_{x} Z_{m k}, \\
M \partial_{t} Z_{m k}+K \partial_{x} Z_{m k}=\nabla_{z} S\left(Z_{m k}\right),
\end{gathered}
$$

where the notation used is as follows: $Z_{m k} \approx z\left(c_{m} \Delta x, d_{k} \Delta t\right), z_{m}^{\mathbf{0}} \approx z\left(c_{m} \Delta x, 0\right)$, $\partial_{t} Z_{m j} \approx \partial_{t} z\left(c_{m} \Delta x, d_{k} \Delta t\right), \partial_{x} Z_{m j} \approx \partial_{x} z\left(c_{m} \Delta x, d_{k} \Delta t\right), z_{m}^{\mathbf{1}} \approx z\left(c_{m} \Delta x, \Delta t\right) . z_{\mathbf{0}}^{k} \approx$ $z\left(0, d_{k} \Delta t\right), z_{1}^{k} \approx z\left(\Delta x, d_{k} \Delta t\right)$, and

$$
c_{m}=\sum_{n=1}^{s} \tilde{a}_{m n}, \quad d_{k}=\sum_{j=1}^{r} a_{k j} .
$$

Corresponding variational equations to (1.7)-(1.11), respectively, are

$$
\begin{gathered}
d Z_{m k}=d z_{m}^{\mathbf{0}}+\Delta t \sum_{j=1}^{r} a_{k j} d\left(\partial_{t} Z_{m j}\right), \\
d z_{m}^{\mathbf{1}}=d z_{m}^{\mathbf{0}}+\Delta t \sum_{k=1}^{r} b_{k} d\left(\partial_{t} Z_{m k}\right), \\
d Z_{m k}=d z_{\mathbf{0}}^{k}+\Delta x \sum_{n=1}^{s} \tilde{a}_{m n} d\left(\partial_{x} Z_{n k}\right), \\
d z_{\mathbf{1}}^{k}=d z_{\mathbf{0}}^{k}+\Delta x \sum_{m=1}^{s} \tilde{b}_{m} d\left(\partial_{x} Z_{m k}\right), \\
M \partial_{t} d Z_{m k}+K \partial_{x} d Z_{m k}=D_{z z} S\left(Z_{m k}\right) d Z_{m k},
\end{gathered}
$$

where $D_{z z} S\left(Z_{m k}\right)$ is a symmetric matrix.

Theorem 1.2. If in the method (1.7)-1.11)

$$
\begin{aligned}
b_{k} b_{j}-b_{k} a_{k j}-b_{j} a_{j k} & =0 \\
\text { and } \quad \tilde{b}_{m} \tilde{b}_{n}-\tilde{b}_{m} \tilde{a}_{m n}-\tilde{b}_{n} \tilde{a}_{n m} & =0
\end{aligned}
$$

hold for $k, j=1,2, \ldots, r$ and $m, n=1,2, \ldots, s$, then the method (1.7) (1.11) is multi-symplectic with the conservation law

$$
\begin{aligned}
& \Delta x \sum_{m=1}^{s} \tilde{b}_{m}\left(\left(\widetilde{d z_{m}^{\mathbf{1}}}\right)^{T} M^{T}\left(d z_{m}^{\mathbf{1}}\right)-\left(\widetilde{d z_{m}^{\mathbf{0}}}\right)^{T} M^{T}\left(d z_{m}^{\mathbf{0}}\right)\right) \\
& \left.+\Delta t \sum_{k=1}^{r} b_{k}\left(\widetilde{\left(d z_{\mathbf{1}}^{k}\right.}\right)^{T} K^{T}\left(d z_{\mathbf{1}}^{k}\right)-\left(\widetilde{d z_{\mathbf{0}}^{k}}\right)^{T} K^{T}\left(d z_{\mathbf{0}}^{k}\right)\right)=0,
\end{aligned}
$$

where

$$
\begin{array}{r}
\left\{\widetilde{d z_{m}^{\mathbf{1}}}, \widetilde{d z_{m}^{\mathbf{0}}}, \widetilde{d z_{\mathbf{1}}^{k}}, \widetilde{d z_{\mathbf{0}}^{k}}, \widetilde{d Z_{m k}}, d\left(\widetilde{\partial_{x} Z_{m k}}\right), d\left(\widetilde{\partial_{t} Z_{m k}}\right)\right\} \\
\text { and } \quad\left\{d z_{m}^{\mathbf{1}}, d z_{m}^{\mathbf{0}}, d z_{\mathbf{1}}^{k}, d z_{\mathbf{0}}^{k}, d Z_{m k}, d\left(\partial_{x} Z_{m k}\right), d\left(\partial_{t} Z_{m k}\right)\right\}
\end{array}
$$

are solutions of the variational equation (1.12)-(1.16). 
Proof. Let

$$
\begin{aligned}
& \left\{\widetilde{d z_{m}^{1}}, \widetilde{d z_{m}^{0}}, \widetilde{d z_{1}^{k}}, \widetilde{d z_{0}^{k}}, \widetilde{d Z_{m k}}, d\left(\widetilde{\partial_{x} Z_{m k}}\right), d\left(\widetilde{\partial_{t} Z_{m k}}\right)\right\}, \\
& \left\{d z_{m}^{1}, d z_{m}^{0}, d z_{1}^{k}, d z_{\mathbf{0}}^{k}, d Z_{m k}, d\left(\partial_{x} Z_{m k}\right), d\left(\partial_{t} Z_{m k}\right)\right\}
\end{aligned}
$$

be solutions of the variational equation (1.12)-(1.16). It follows from (1.12)-(1.16) and (1.17) -1.18) that

$$
\begin{aligned}
& \left(\widetilde{d z_{m}^{\mathbf{1}}}\right)^{T} M^{T}\left(d z_{m}^{\mathbf{1}}\right)-\left(\widetilde{d z_{m}^{\mathbf{0}}}\right)^{T} M^{T}\left(d z_{m}^{\mathbf{0}}\right) \\
= & \Delta t \sum_{k=1}^{r} b_{k}\left(d \widetilde{\left(\widetilde{\partial_{t} Z_{m k}}\right)^{T}} M^{T}\left(d Z_{m k}\right)+\left(\widetilde{d Z_{m k}}\right)^{T} M^{T} d\left(\partial_{t} Z_{m k}\right)\right) \\
& +(\Delta t)^{2} \sum_{j, k=1}^{r}\left(b_{k} b_{j}-b_{k} a_{k j}-b_{j} a_{j k}\right) d\left(\widetilde{\partial_{t} Z_{m k}}\right)^{T} M^{T} d\left(\partial_{t} Z_{m k}\right) \\
= & \Delta t \sum_{k=1}^{r} b_{k}\left(d\left(\widetilde{\partial_{t} Z_{m k}}\right)^{T} M^{T}\left(d Z_{m k}\right)+\left(\widetilde{d Z_{m k}}\right)^{T} M^{T} d\left(\partial_{t} Z_{m k}\right)\right)
\end{aligned}
$$

and

$$
\begin{aligned}
& \left.\left(\widetilde{d z_{\mathbf{1}}^{k}}\right)^{T} K^{T}\left(d z_{\mathbf{1}}^{k}\right)-\widetilde{\left(d z_{\mathbf{0}}^{k}\right.}\right)^{T} K^{T}\left(d z_{\mathbf{0}}^{k}\right) \\
& =\Delta x \sum_{m=1}^{s} \tilde{b}_{m}\left(d\left(\widetilde{\partial_{x} Z_{m k}}\right)^{T} K^{T}\left(d Z_{m k}\right)+\left(\widetilde{d Z_{m k}}\right)^{T} K^{T} d\left(\partial_{x} Z_{m k}\right)\right) .
\end{aligned}
$$

Using (1.16) and the symmetry of the matrix $D_{z z} S\left(Z_{m k}\right)$ produces

$$
\begin{aligned}
& d\left(\widetilde{\partial_{t} Z_{m k}}\right)^{T} M^{T}\left(d Z_{m k}\right)+\left(\widetilde{d Z_{m k}}\right)^{T} M^{T} d\left(\partial_{t} Z_{m k}\right) \\
& +d\left(\widetilde{\partial_{x} Z_{m k}}\right)^{T} K^{T}\left(d Z_{m k}\right)+\left(\widetilde{d Z_{m k}}\right)^{T} K^{T} d\left(\partial_{x} Z_{m k}\right)=0 .
\end{aligned}
$$

Combining (1.20), (1.21), and (1.22), the proof of the theorem is completed.

Remark 1.3. This theorem can be extended to the Hamiltonian partial differential equation with varying coefficients

$$
M(x) z_{t}+K(t) z_{x}=\nabla_{z} S(z, x, t),
$$

where $M(x)$ and $K(t)$ are skew-symmetric matrices and smooth in $x$ and $t$, respectively, and $S(z, x, t)$ is a smooth real function.

The following corollary is a natural extension of the result in $[9]$.

Corollary 1.4. If in (1.7)-11.11), the method applied to both the time direction and the space direction is Gauss-Legendre, then the method (1.7)-(1.11) is a multisymplectic integrator.

\section{Partitioned Runge-Kutta methods}

We consider the blocked Hamiltonian partial differential equation

$$
\left(\begin{array}{cc}
M_{1} & M_{0} \\
-M_{0}^{T} & M_{2}
\end{array}\right)\left(\begin{array}{c}
p_{t} \\
q_{t}
\end{array}\right)+\left(\begin{array}{cc}
K_{1} & K_{0} \\
-K_{0}^{T} & K_{2}
\end{array}\right)\left(\begin{array}{c}
p_{x} \\
q_{x}
\end{array}\right)=\left(\begin{array}{c}
\nabla_{p} S(p, q) \\
\nabla_{q} S(p, q)
\end{array}\right),
$$

where $M_{\tau}, K_{\tau}(\tau=1,2)$ are $\alpha \times \alpha$ skew-symmetric matrices, $M_{0}, K_{0}$ are $\alpha \times \alpha$ matrices, and $S(p, q)$ is a smooth real function in $p=\left(p_{1}, p_{2}, \ldots, p_{\alpha}\right)^{T}$ and $q=\left(q_{1}, q_{2}, \ldots, q_{\alpha}\right)^{T}$. 
The corresponding multi-symplectic conservation law is

$$
\frac{\partial \omega(U, V)}{\partial t}+\frac{\partial \kappa(U, V)}{\partial x}=0
$$

where

$$
\omega(U, V)=U^{T}\left(\begin{array}{cc}
M_{1} & M_{0} \\
-M_{0}^{T} & M_{2}
\end{array}\right)^{T} V, \quad \kappa(U, V)=U^{T}\left(\begin{array}{cc}
K_{1} & K_{0} \\
-K_{0}^{T} & K_{2}
\end{array}\right)^{T} V,
$$

$U(x, t)$ and $V(x, t)$ are solutions of the variational equation

$$
\left(\begin{array}{cc}
M_{1} & M_{0} \\
-M_{0}^{T} & M_{2}
\end{array}\right) d z_{t}+\left(\begin{array}{cc}
K_{1} & K_{0} \\
-K_{0}^{T} & K_{2}
\end{array}\right) d z_{x}=D_{z z} S(z) d z
$$

and $z=\left(p_{1}, p_{2}, \ldots, p_{\alpha}, q_{1}, q_{2}, \ldots, q_{\alpha}\right)^{T}$. Now we apply the partitioned Runge-Kutta method to the equation (2.1):

$$
\begin{aligned}
& P_{m k}=p_{m}^{\mathbf{0}}+\Delta t \sum_{j=1}^{r} a_{k j}^{(1)} \partial_{t} P_{m j}, \\
& Q_{m k}=q_{m}^{\mathbf{0}}+\Delta t \sum_{j=1}^{r} a_{k j}^{(2)} \partial_{t} Q_{m j}, \\
& p_{m}^{\mathbf{1}}=p_{m}^{\mathbf{0}}+\Delta t \sum_{k=1}^{r} b_{k}^{(1)} \partial_{t} P_{m k}, \\
& q_{m}^{\mathbf{1}}=q_{m}^{\mathbf{0}}+\Delta t \sum_{k=1}^{r} b_{k}^{(2)} \partial_{t} Q_{m k}, \\
& P_{m k}=p_{\mathbf{0}}^{k}+\Delta x \sum_{n=1}^{s} \tilde{a}_{m n}^{(1)} \partial_{x} P_{n k}, \\
& Q_{m k}=q_{\mathbf{0}}^{k}+\Delta x \sum_{n=1}^{s} \tilde{a}_{m n}^{(2)} \partial_{x} Q_{n k}, \\
& p_{\mathbf{1}}^{k}=p_{\mathbf{0}}^{k}+\Delta x \sum_{m=1}^{s} \tilde{b}_{m}^{(1)} \partial_{x} P_{m k}, \\
& q_{\mathbf{1}}^{k}=q_{\mathbf{0}}^{k}+\Delta x \sum_{m=1}^{s} \tilde{b}_{m}^{(2)} \partial_{x} Q_{m k}, \\
& \left(\begin{array}{cc}
M_{1} & M_{0} \\
-M_{0}^{T} & M_{2}
\end{array}\right)\left(\begin{array}{c}
\partial_{t} P_{m k} \\
\partial_{t} Q_{m k}
\end{array}\right)+\left(\begin{array}{cc}
K_{1} & K_{0} \\
-K_{0}^{T} & K_{2}
\end{array}\right)\left(\begin{array}{c}
\partial_{x} P_{m k} \\
\partial_{x} Q_{m k}
\end{array}\right) \\
& =\left(\begin{array}{c}
\nabla_{p} S\left(P_{m k}, Q_{m k}\right) \\
\nabla_{q} S\left(P_{m k}, Q_{m k}\right)
\end{array}\right),
\end{aligned}
$$

where we make use of the notation

$$
\begin{array}{lr}
p_{m}^{\mathbf{0}} \approx p\left(c_{m} \Delta x, 0\right), & p_{m}^{\mathbf{1}} \approx p\left(c_{m} \Delta x, \Delta t\right), \\
p_{\mathbf{0}}^{k} \approx p\left(0, d_{k} \Delta t\right), & p_{\mathbf{1}}^{k} \approx p\left(\Delta x, d_{k} \Delta t\right), \\
q_{m}^{\mathbf{0}} \approx q\left(c_{m} \Delta x, 0\right), & q_{m}^{\mathbf{1}} \approx q\left(c_{m} \Delta x, \Delta t\right), \\
q_{\mathbf{0}}^{k} \approx q\left(0, d_{k} \Delta t\right), & q_{\mathbf{1}}^{k} \approx q\left(\Delta x, d_{k} \Delta t\right), \\
P_{m k} \approx p\left(c_{m} \Delta x, d_{k} \Delta t\right), & Q_{m k} \approx q\left(c_{m} \Delta x, d_{k} \Delta t\right), \\
\partial_{t} P_{m k} \approx \frac{\partial p}{\partial t}\left(c_{m} \Delta x, d_{k} \Delta t\right), & \partial_{x} P_{m k} \approx \frac{\partial p}{\partial x}\left(c_{m} \Delta x, d_{k} \Delta t\right), \\
\partial_{t} Q_{m k} \approx \frac{\partial q}{\partial t}\left(c_{m} \Delta x, d_{k} \Delta t\right), & \partial_{x} Q_{m k} \approx \frac{\partial q}{\partial x}\left(c_{m} \Delta x, d_{k} \Delta t\right)
\end{array}
$$

under the assumption that

$$
\sum_{n=1}^{s} \tilde{a}_{m n}^{(1)}=\sum_{n=1}^{s} \tilde{a}_{m n}^{(2)}=c_{m}, \quad \sum_{j=1}^{r} a_{k j}^{(1)}=\sum_{j=1}^{r} a_{k j}^{(2)}=d_{k} .
$$


The system of variational equations of the method (2.4)-(2.12) corresponding to (1.6) is

$$
\begin{aligned}
& d P_{m k}=d p_{m}^{\mathbf{0}}+\Delta t \sum_{j=1}^{r} a_{k j}^{(1)} d \partial_{t} P_{m j}, \\
& d Q_{m k}=d q_{m}^{\mathbf{0}}+\Delta t \sum_{j=1}^{r} a_{k j}^{(2)} d \partial_{t} Q_{m j}, \\
& d p_{m}^{\mathbf{1}}=d p_{m}^{\mathbf{0}}+\Delta t \sum_{k=1}^{r} b_{k}^{(1)} d \partial_{t} P_{m k}, \\
& d q_{m}^{\mathbf{1}}=d q_{m}^{\mathbf{0}}+\Delta t \sum_{k=1}^{r} b_{k}^{(2)} d \partial_{t} Q_{m k}, \\
& d P_{m k}=d p_{\mathbf{0}}^{k}+\Delta x \sum_{n=1}^{s} \tilde{a}_{m n}^{(1)} d \partial_{x} P_{n k}, \\
& d Q_{m k}=d q_{\mathbf{0}}^{k}+\Delta x \sum_{n=1}^{s} \tilde{a}_{m n}^{(2)} d \partial_{x} Q_{n k}, \\
& d p_{\mathbf{1}}^{k}=d p_{\mathbf{0}}^{k}+\Delta x \sum_{m=1}^{s} \tilde{b}_{m}^{(1)} d \partial_{x} P_{m k}, \\
& d q_{\mathbf{1}}^{k}=d q_{\mathbf{0}}^{k}+\Delta x \sum_{m=1}^{s} \tilde{b}_{m}^{(2)} d \partial_{x} Q_{m k}, \\
& M d\left(\partial_{t} Z_{m k}\right)+K d\left(\partial_{x} Z_{m k}\right)=A_{m k} d\left(Z_{m k}\right),
\end{aligned}
$$

where

$$
\begin{aligned}
& d\left(Z_{m k}\right)=\left(\begin{array}{c}
d P_{m k} \\
d Q_{m k}
\end{array}\right) \\
& d\left(\partial_{t} Z_{m k}\right)=\left(\begin{array}{c}
d \partial_{t} P_{m k} \\
d \partial_{t} Q_{m k}
\end{array}\right), \\
& d\left(\partial_{x} Z_{m k}\right)=\left(\begin{array}{c}
d \partial_{x} P_{m k} \\
d \partial_{x} Q_{m k}
\end{array}\right) \\
& A_{m k}=\left(\begin{array}{cc}
D_{p p} S\left(P_{m k}, Q_{m k}\right) & D_{p q} S\left(P_{m k}, Q_{m k}\right) \\
D_{q p} S\left(P_{m k}, Q_{m k}\right) & D_{q q} S\left(P_{m k}, Q_{m k}\right)
\end{array}\right) \text {, } \\
& M=\left(\begin{array}{cc}
M_{1} & M_{0} \\
-M_{0}^{T} & M_{2}
\end{array}\right), \quad K=\left(\begin{array}{cc}
K_{1} & K_{0} \\
-K_{0}^{T} & K_{2}
\end{array}\right) \text {. }
\end{aligned}
$$

Obviously, $A_{m k}$ is a symmetric matrix. Now we let

$$
\begin{aligned}
& \left\{d p_{m}^{\mathbf{1}}, d p_{m}^{\mathbf{0}}, d p_{\mathbf{1}}^{k}, d p_{\mathbf{0}}^{k}, d P_{m k}, d \partial_{t} P_{m k}, d \partial_{x} P_{m k},\right. \\
& \left.d q_{m}^{\mathbf{1}}, d q_{m}^{\mathbf{0}}, d q_{\mathbf{1}}^{k}, d q_{\mathbf{0}}^{k}, d Q_{m k}, d \partial_{t} Q_{m k}, d \partial_{x} Q_{m k}\right\}, \\
& \left\{\widetilde{d p_{m}^{\mathbf{1}}}, \widetilde{d p_{m}^{\mathbf{0}}}, \widetilde{d p_{\mathbf{1}}^{k}}, \widetilde{d p_{\mathbf{0}}^{k}}, \widetilde{d P_{m k}}, \widetilde{d \partial_{t} P_{m k}}, \widetilde{d \partial_{x} P_{m k}},\right. \\
& \left.\widetilde{d q_{m}^{\mathbf{1}}}, \widetilde{d q_{m}^{\mathbf{0}}}, \widetilde{d q_{\mathbf{1}}^{k}}, \widetilde{d q_{\mathbf{0}}^{k}}, \widetilde{d Q_{m k}}, \widetilde{d \partial_{t} Q_{m k}}, \widetilde{d \partial_{x} Q_{m k}}\right\}
\end{aligned}
$$


be solutions of the variational equations (2.14) $-(2.22)$, and

$$
\begin{aligned}
\delta_{t} \omega_{m} & =\left({\widetilde{d p_{m}^{\mathbf{1}}}}^{T},{\widetilde{d q_{m}^{\mathbf{1}}}}^{T}\right) M^{T}\left(\begin{array}{c}
d p_{m}^{\mathbf{1}} \\
d q_{m}^{\mathbf{1}}
\end{array}\right)-\left({\widetilde{d p_{m}^{\mathbf{0}}}}^{T},{\widetilde{d q_{m}^{\mathbf{0}}}}^{T}\right) M^{T}\left(\begin{array}{c}
d p_{m}^{\mathbf{0}} \\
d q_{m}^{\mathbf{0}}
\end{array}\right), \\
\delta_{x} \kappa^{k} & =\left({\widetilde{d p_{\mathbf{1}}^{k}}}^{T},{\widetilde{d q_{\mathbf{1}}^{k}}}^{T}\right) K^{T}\left(\begin{array}{c}
d p_{1}^{k} \\
d q_{\mathbf{1}}^{k}
\end{array}\right)-\left({\widetilde{d p_{\mathbf{0}}^{k}}}^{T},{\widetilde{d q_{\mathbf{0}}^{k}}}^{T}\right) K^{T}\left(\begin{array}{c}
d p_{\mathbf{0}}^{k} \\
d q_{\mathbf{0}}^{k}
\end{array}\right) .
\end{aligned}
$$

By a straightforward calculation, we have

$$
\begin{aligned}
& \delta_{t} \omega_{m}=\Delta t \sum_{k=1}^{r}\left({\widetilde{d P_{m k}}}^{T},{\widetilde{d Q_{m k}}}^{T}\right) M^{T}\left(\begin{array}{c}
b_{k}^{(1)} d \partial_{t} P_{m k} \\
b_{k}^{(2)} d \partial_{t} Q_{m k}
\end{array}\right) \\
& \left.+\left(b_{k}^{(1)}{\widetilde{d \partial_{t} P_{m k}}}^{T}, b_{k}^{(2)}{\widetilde{d \partial_{t} Q_{m k}}}^{T}\right) M^{T}\left(\begin{array}{c}
d P_{m k} \\
d Q_{m k}
\end{array}\right)\right) \\
& +(\Delta t)^{2} \sum_{j, k=1}^{r}\left(\left(b_{k}^{(1)} a_{k j}^{(1)}+b_{j}^{(1)} a_{j k}^{(1)}-b_{k}^{(1)} b_{j}^{(1)}\right) d\left(\widetilde{\partial_{t} P_{m j}}\right)^{T} M_{1} d\left(\partial_{t} P_{m k}\right)\right. \\
& +\left(b_{k}^{(2)} a_{k j}^{(2)}+b_{j}^{(2)} a_{j k}^{(2)}-b_{k}^{(2)} b_{j}^{(2)}\right) d\left(\widetilde{\partial_{t} Q_{m j}}\right)^{T} M_{2} d\left(\partial_{t} Q_{m k}\right) \\
& +\left(b_{k}^{(2)} a_{k j}^{(1)}+b_{j}^{(1)} a_{j k}^{(2)}-b_{k}^{(2)} b_{j}^{(1)}\right) d\left(\widetilde{\partial_{t} P_{m j}}\right)^{T} M_{0} d\left(\partial_{t} Q_{m k}\right) \\
& \left.+\left(b_{j}^{(2)} b_{k}^{(1)}-b_{j}^{(2)} a_{j k}^{(1)}-b_{k}^{(1)} a_{k j}^{(2)}\right) d\left(\widetilde{\partial_{t} Q_{m j}}\right)^{T} M_{0} d\left(\partial_{t} P_{m k}\right)\right)
\end{aligned}
$$

and

$$
\begin{aligned}
\delta_{x} \kappa^{k}=\Delta x \sum_{m=1}^{s}( & \left({\widetilde{d P_{m k}}}^{T},{\widetilde{d Q_{m k}}}^{T}\right) K^{T}\left(\begin{array}{c}
\tilde{b}_{k}^{(1)} d \partial_{x} P_{m k} \\
\tilde{b}_{k}^{(2)} d \partial_{x} Q_{m k}
\end{array}\right) \\
& \left.+\left(\tilde{b}_{k}^{(1)} d{\widetilde{\partial \partial_{x} P_{m k}}}^{T}, \tilde{b}_{k}^{(2)} d{\widetilde{\partial \partial_{x} Q_{m k}}}^{T}\right) K^{T}\left(\begin{array}{c}
d P_{m k} \\
d Q_{m k}
\end{array}\right)\right) \\
& +(\Delta x)^{2} \sum_{m, n=1}^{s}\left(\left(\tilde{b}_{m}^{(1)} \tilde{a}_{m n}^{(1)}+\tilde{b}_{n}^{(1)} \tilde{a}_{n m}^{(1)}-\tilde{b}_{m}^{(1)} \tilde{b}_{n}^{(1)}\right) d\left(\widetilde{\partial_{x} P_{n k}}\right)^{T} K_{1} d\left(\partial_{x} P_{m k}\right)\right. \\
& \left.+\left(\tilde{b}_{m}^{(2)} \tilde{a}_{m n}^{(2)}+\tilde{b}_{n}^{(2)} \tilde{a}_{n m}^{(2)}-\tilde{b}_{m}^{(2)} \tilde{b}_{n}^{(2)}\right) d \widetilde{\left(\widetilde{\partial x}_{x} Q_{n k}\right.}\right)^{T} K_{2} d\left(\partial_{x} Q_{m k}\right) \\
& \left.+\left(\tilde{b}_{m}^{(2)} \tilde{a}_{m n}^{(1)}+\tilde{b}_{n}^{(1)} \tilde{a}_{n m}^{(2)}-\tilde{b}_{m}^{(2)} \tilde{b}_{n}^{(1)}\right) d \widetilde{\left(\partial_{x} P_{n k}\right.}\right)^{T} K_{0} d\left(\partial_{x} Q_{m k}\right) \\
& +\left(\tilde{b}_{n}^{(2)} \tilde{b}_{m}^{(1)}-\tilde{b}_{m}^{(1)} \tilde{a}_{m n}^{(2)}-\tilde{b}_{n}^{(2)} \tilde{a}_{n m}^{(1)}\right) d\left(\widetilde{\partial_{x} Q_{n k}}\right)^{T} K_{0} d\left(\partial_{x} P_{m k}\right) .
\end{aligned}
$$

If for $k=1,2, \ldots, r$ and $m=1,2, \ldots, s$,

$$
b_{k}^{(1)}=b_{k}^{(2)}=b_{k}, \quad \tilde{b}_{m}^{(1)}=\tilde{b}_{m}^{(2)}=\tilde{b}_{m},
$$

then the multi-symplectic conservation law of the method (2.4)-(2.12) corresponding to (1.5) is

$$
\Delta x \sum_{m=1}^{s} \tilde{b}_{m} \delta_{t} \omega_{m}+\Delta t \sum_{k=1}^{r} b_{k} \delta_{x} \kappa^{k}=0
$$


Consequently, in this case, it is sufficient for (2.28), which holds, that

$$
I_{1}=0 \quad \text { and } \quad I_{2}=0 \text {, }
$$

where

$$
\begin{aligned}
I_{1}=(\Delta t)^{2} \sum_{j, k=1}^{r}( & \left.\left(b_{k}^{(1)} a_{k j}^{(1)}+b_{j}^{(1)} a_{j k}^{(1)}-b_{k}^{(1)} b_{j}^{(1)}\right) d \widetilde{d\left(\widetilde{\partial}_{t} P_{m j}\right.}\right)^{T} M_{1} d\left(\partial_{t} P_{m k}\right) \\
& +\left(b_{k}^{(2)} a_{k j}^{(2)}+b_{j}^{(2)} a_{j k}^{(2)}-b_{k}^{(2)} b_{j}^{(2)}\right) d\left(\widetilde{\partial_{t} Q_{m j}}\right)^{T} M_{2} d\left(\partial_{t} Q_{m k}\right) \\
& +\left(b_{k}^{(2)} a_{k j}^{(1)}+b_{j}^{(1)} a_{j k}^{(2)}-b_{k}^{(2)} b_{j}^{(1)}\right) d\left(\widetilde{\partial_{t} P_{m j}}\right)^{T} M_{0} d\left(\partial_{t} Q_{m k}\right) \\
& \left.+\left(b_{j}^{(2)} b_{k}^{(1)}-b_{j}^{(2)} a_{j k}^{(1)}-b_{k}^{(1)} a_{k j}^{(2)}\right) d\left(\widetilde{\partial_{t} Q_{m j}}\right)^{T} M_{0} d\left(\partial_{t} P_{m k}\right)\right)
\end{aligned}
$$

and

$$
\begin{aligned}
I_{2}=(\Delta x)^{2} \sum_{m, n=1}^{s}( & \left(\tilde{b}_{m}^{(1)} \tilde{a}_{m n}^{(1)}+\tilde{b}_{n}^{(1)} \tilde{a}_{n m}^{(1)}-\tilde{b}_{m}^{(1)} \tilde{b}_{n}^{(1)}\right) d \widetilde{\left(\widetilde{\partial x}_{x} P_{n k}\right)^{T}} K_{1} d\left(\partial_{x} P_{m k}\right) \\
& +\left(\tilde{b}_{m}^{(2)} \tilde{a}_{m n}^{(2)}+\tilde{b}_{n}^{(2)} \tilde{a}_{n m}^{(2)}-\tilde{b}_{m}^{(2)} \tilde{b}_{n}^{(2)}\right) d\left(\widetilde{\partial_{x} Q_{n k}}\right)^{T} K_{2} d\left(\partial_{x} Q_{m k}\right) \\
& +\left(\tilde{b}_{m}^{(2)} \tilde{a}_{m n}^{(1)}+\tilde{b}_{n}^{(1)} \tilde{a}_{n m}^{(2)}-\tilde{b}_{m}^{(2)} \tilde{b}_{n}^{(1)}\right) d\left(\widetilde{\partial_{x} P_{n k}}\right)^{T} K_{0} d\left(\partial_{x} Q_{m k}\right) \\
& \left.+\left(\tilde{b}_{n}^{(2)} \tilde{b}_{m}^{(1)}-\tilde{b}_{m}^{(1)} \tilde{a}_{m n}^{(2)}-\tilde{b}_{n}^{(2)} \tilde{a}_{n m}^{(1)}\right) d\left(\widetilde{\partial_{x} Q_{n k}}\right)^{T} K_{0} d\left(\partial_{x} P_{m k}\right)\right)
\end{aligned}
$$

We let

$$
\begin{aligned}
\left(\mu_{1}\right)_{k j} & =b_{k}^{(1)} a_{k j}^{(1)}+b_{j}^{(1)} a_{j k}^{(1)}-b_{k}^{(1)} b_{j}^{(1)}, \\
\left(\mu_{2}\right)_{k j} & =b_{k}^{(2)} a_{k j}^{(2)}+b_{j}^{(2)} a_{j k}^{(2)}-b_{k}^{(2)} b_{j}^{(2)}, \\
\left(\mu_{3}\right)_{k j} & =b_{k}^{(2)} a_{k j}^{(1)}+b_{j}^{(1)} a_{j k}^{(2)}-b_{k}^{(2)} b_{j}^{(1)}, \\
\left(\nu_{1}\right)_{m n} & =\tilde{b}_{m}^{(1)} \tilde{a}_{m n}^{(1)}+\tilde{b}_{n}^{(1)} \tilde{a}_{n m}^{(1)}-\tilde{b}_{m}^{(1)} \tilde{b}_{n}^{(1)}, \\
\left(\nu_{2}\right)_{m n} & =\tilde{b}_{m}^{(2)} \tilde{a}_{m n}^{(2)}+\tilde{b}_{n}^{(2)} \tilde{a}_{n m}^{(2)}-\tilde{b}_{m}^{(2)} \tilde{b}_{n}^{(2)}, \\
\left(\nu_{3}\right)_{m n} & =\tilde{b}_{m}^{(2)} \tilde{a}_{m n}^{(1)}+\tilde{b}_{n}^{(1)} \tilde{a}_{n m}^{(2)}-\tilde{b}_{m}^{(2)} \tilde{b}_{n}^{(1)} .
\end{aligned}
$$

Then this leads to the following result.

Theorem 2.1. In the method (2.4)-(2.12), suppose that (2.13) and (2.27) hold. The method (2.4)-(2.12) is multi-symplectic, with discrete multi-symplectic law (2.28), if one of following conditions holds.

(1) for $\tau=1,2,3$,

$$
\begin{aligned}
& \left(\mu_{\tau}\right)_{k j}=0(k, j=1,2, \ldots, r) \quad \text { and } \quad\left(\nu_{\tau}\right)_{m n}=0(m, n=1,2, \ldots, s), \\
& \text { when } M_{\lambda} \neq 0, K_{\lambda} \neq 0(\lambda=1,2), M_{0} \neq 0 \text { and } K_{0} \neq 0 ;
\end{aligned}
$$


(2) for $\tau=1,2,3$,

$$
\begin{gathered}
\left(\mu_{1}\right)_{k j}=\left(\mu_{2}\right)_{k j}=0(k, j=1,2, \ldots, r) \\
\left(\text { resp. }\left(\mu_{\tau}\right)_{k j}=0(k, j=1,2, \ldots, r)\right), \\
\left(\nu_{\tau}\right)_{m n}=0(m, n=1,2, \ldots, s) \\
\left(\text { resp. }\left(\nu_{1}\right)_{m n}=\left(\nu_{2}\right)_{m n}=0(m, n=1,2, \ldots, s)\right),
\end{gathered}
$$

when $M_{0}=0\left(\right.$ resp. $\left.K_{0}=0\right)$;

(3) for $\tau=1,2,\left(\mu_{\tau}\right)_{k j}=0(k, j=1,2, \ldots, r)$ and $\left(\nu_{\tau}\right)_{m n}=0(m, n=$ $1,2, \ldots, s)$, when $M_{0}=0$ and $K_{0}=0$;

(4) $\left(\mu_{3}\right)_{k j}=\left(\nu_{3}\right)_{m n}=0$, for $k, j=1,2, \ldots, r, m, n=1,2, \ldots, s$, when $M_{\tau}=$ $K_{\tau}=0$ for $\tau=1,2$ (this is a typical multi-symplectic partitioned condition);

(5) for $\tau=1,2,\left(\mu_{3}\right)_{k j}=\left(\nu_{\tau}\right)_{m n}=0$, for $k, j=1,2, \ldots, r, m, n=1,2, \ldots, s$, when $M_{\sigma}=K_{0}=0$ for $\sigma=1,2$;

(6) for $\tau=1,2,\left(\mu_{\tau}\right)_{k j}=\left(\nu_{3}\right)_{m n}=0$, for $k, j=1,2, \ldots, r, m, n=1,2, \ldots, s$, when $M_{0}=K_{\sigma}=0$ for $\sigma=1,2$;

(7) $\left(\mu_{1}\right)_{k j}=\left(\nu_{3}\right)_{m n}=0$, for $k, j=1,2, \ldots, r, m, n=1,2, \ldots, s$, when $M_{0}=$ $M_{2}=K_{\sigma}=0$ for $\sigma=1,2$

(8) $\left(\mu_{3}\right)_{k j}=\left(\nu_{1}\right)_{m n}=0$, for $k, j=1,2, \ldots, r, m, n=1,2, \ldots, s$, when $M_{\sigma}=$ $K_{0}=K_{2}=0$ for $\sigma=1,2$.

Now we give some remarks.

Remark 2.2. In Theorem 2.1 we list only eight conditions for multi-symplecticity of the partitioned Runge-Kutta method of (2.4)-2.12). By using $I_{1}=0$ and $I_{2}=0$, we can conclude more conditions for multi-symplectic partitioned RungeKutta methods. This theorem can be extended naturally to the case of Hamiltonian partial differential equations with varying coefficients.

Remark 2.3. It is trivial and apparent to extend Theorem 1.2 and Theorem 2.1 to the Hamiltonian partial differential equation with higher spatial dimension

$$
M z_{t}+\sum_{\tau=1}^{\iota} K_{\tau} z_{x_{\tau}}=\nabla_{z} S(z)
$$

where $\iota \geq 2, M$ and $K_{\tau}(\tau=1,2, \ldots, \iota)$ are skew-symmetric matrices, and $S$ is a smooth function.

Remark 2.4. In Theorem 2.1 the condition (1) implies $a_{k j}^{(1)}=a_{k j}^{(2)}$ for $k, j=1,2, \ldots r$ and $\tilde{a}_{m n}^{(1)}=\tilde{a}_{m n}^{(2)}$ for $m, n=1,2, \ldots, s$. In fact, in this case only one symplectic Runge-Kutta method is applied in each direction.

Remark 2.5. Consider the nonlinear Schrödinger equation

$$
i \frac{\partial \psi}{\partial t}+\frac{\partial^{2} \psi}{\partial x^{2}}+|\psi|^{2} \psi=0
$$

Let $\psi(x, t)=u(x, t)+i v(x, t)$. Then the equation (2.36) is read as

$$
\left\{\begin{array}{c}
-\frac{\partial v}{\partial t}+\frac{\partial^{2} u}{\partial x^{2}}+\left(u^{2}+v^{2}\right) u=0 \\
\frac{\partial u}{\partial t}+\frac{\partial^{2} v}{\partial x^{2}}+\left(u^{2}+v^{2}\right) v=0 .
\end{array}\right.
$$

We take $z=\left(u, v, u_{x}, v_{x}\right)^{T}$. Then the equation (2.37) can be rewritten as

$$
M \frac{\partial z}{\partial t}+K \frac{\partial z}{\partial x}=\nabla_{z} S(z, t),
$$


where

$$
M=\left(\begin{array}{cccc}
0 & -1 & 0 & 0 \\
1 & 0 & 0 & 0 \\
0 & 0 & 0 & 0 \\
0 & 0 & 0 & 0
\end{array}\right), \quad K=\left(\begin{array}{cccc}
0 & 0 & 1 & 0 \\
0 & 0 & 0 & 1 \\
-1 & 0 & 0 & 0 \\
0 & -1 & 0 & 0
\end{array}\right)
$$

and

$$
S(z, t)=-\frac{1}{4}\left(u^{2}+v^{2}\right)^{2}-\frac{1}{2}\left(u_{x}^{2}+v_{x}^{2}\right) .
$$

The equation (2.38) is in accordance with the case of condition (7) in Theorem 2.1. Thus the partitioned Runge-Kutta (2.4)-(2.12) method can be applied to the equation (2.38).

Remark 2.6. We consider the nonlinear Dirac equation

$$
\psi_{t}=A \psi_{x}+i f\left(\left|\psi_{1}\right|^{2}-\left|\psi_{2}\right|^{2}\right) B \psi
$$

where $\psi=\left(\psi_{1}, \psi_{2}\right)^{T}, i=\sqrt{-1}, f(s)$ is a real function of a real variable $s$, matrices $A$ and $B$ are $\left(\begin{array}{cc}0 & -1 \\ -1 & 0\end{array}\right)$ and $\left(\begin{array}{cc}-1 & 0 \\ 0 & 1\end{array}\right)$, respectively, and $\varphi=\left(\varphi_{1}, \varphi_{2}\right)^{T}$ is sufficiently smooth. Let $\psi_{j}=u_{j}+i v_{j}(j=1,2)$ and $z=\left(u_{1}, v_{1}, u_{2}, v_{2}\right)^{T}$. Then the equation (2.39) can be written as

$$
M z_{t}+K z_{x}=\nabla_{z} S(z),
$$

where

$$
M=\left(\begin{array}{cccc}
0 & 1 & 0 & 0 \\
-1 & 0 & 0 & 0 \\
0 & 0 & 0 & 1 \\
0 & 0 & -1 & 0
\end{array}\right), \quad K=\left(\begin{array}{cccc}
0 & 0 & 0 & 1 \\
0 & 0 & -1 & 0 \\
0 & 1 & 0 & 0 \\
-1 & 0 & 0 & 0
\end{array}\right)
$$

and

$$
S(z)=\frac{-1}{2} F\left(u_{1}^{2}+v_{1}^{2}-u_{2}^{2}-v_{2}^{2}\right)
$$

where the real smooth function $F(\zeta)$ satisfies $\frac{d}{d \zeta} F(\zeta)=f(\zeta)$.

The equation (2.40) is in the case of condition (6) in Theorem 2.1.

Now denoting $z=\left(u_{1}, u_{2}, v_{1}, v_{2}\right)$, the equation (2.39) can be rewritten as

$$
\hat{M} \hat{z}_{t}+\hat{K} \hat{z}_{x}=\nabla_{\hat{z}} S(\hat{z}),
$$

where

$$
\hat{M}=\left(\begin{array}{cccc}
0 & 0 & 1 & 0 \\
0 & 0 & 0 & 1 \\
-1 & 0 & 0 & 0 \\
0 & -1 & 0 & 0
\end{array}\right), \quad \hat{K}=\left(\begin{array}{cccc}
0 & 0 & 0 & 1 \\
0 & 0 & 1 & 0 \\
0 & -1 & 0 & 0 \\
-1 & 0 & 0 & 0
\end{array}\right)
$$

The equation (2.41) is in the case of condition (4) in Theorem 2.1.

\section{Hamiltonian WAVE EQUATions}

In this section we consider the scalar wave equation

$$
u_{t t}=u_{x x}-G^{\prime}(u), \quad(x, t) \in \Omega \subset R^{2},
$$

where $G: R \rightarrow R$ is a smooth function. The investigation on symplectic integration for the equation (3.1) can be found in [4] and the references therein.

Let $\hat{z}=(u, p, v, w)^{T}, u_{t}=v, u_{x}=w$. Then the equation (3.1) can be written as

$$
M \hat{z}_{t}+K \hat{z}_{x}=\nabla_{\hat{z}} S(\hat{z}),
$$


where

$$
M=\left(\begin{array}{cccc}
0 & 0 & 1 & 0 \\
0 & 0 & 0 & 0 \\
-1 & 0 & 0 & 0 \\
0 & 0 & 0 & 0
\end{array}\right) \quad K=\left(\begin{array}{cccc}
0 & 0 & 0 & -1 \\
0 & 0 & 0 & 0 \\
0 & 0 & 0 & 0 \\
1 & 0 & 0 & 0
\end{array}\right)
$$

The case (4) in Theorem 2.1 is suitable for the equation (3.2).

In [9], the following result is given by Reich.

Proposition 3.1. Let (3.1) be discretized in space and in time by a pair of GaussLegendre collocation methods with stages $s, r$, respectively. Then the resulting discretization is a multi-symplectic integrator.

This result has fundamental importance for the multi-symplectic methods of Hamiltonian PDEs. It implies the possibility of constructing higher-order multisymplectic schemes.

Now we investigate the multi-symplecticity of partitioned Runge-Kutta methods for the equation (3.1) by using the multi-symplectic conservation law (see [9])

$$
\partial_{t}\left(d u \wedge d u_{t}\right)=\partial_{x}\left(d u \wedge d u_{x}\right)
$$

The partitioned Runge-Kutta method applied to the equation (3.1) is

$$
\begin{gathered}
U_{m k}=u_{\mathbf{0}}^{k}+\Delta x \sum_{n=1}^{s} \tilde{a}_{m n}^{(1)} \partial_{x} U_{n k}, \\
W_{m k}=u_{\mathbf{0}}^{k}+\Delta x \sum_{n=1}^{s} \tilde{a}_{m n}^{(2)} \partial_{x} W_{n k}, \\
U_{m k}=u_{m}^{\mathbf{0}}+\Delta t \sum_{j=1}^{r} a_{k j}^{(1)} \partial_{t} U_{m j}, \\
V_{m k}=v_{m}^{\mathbf{0}}+\Delta t \sum_{j=1}^{r} a_{k j}^{(2)} \partial_{t} V_{m j}, \\
u_{\mathbf{1}}^{k}=u_{\mathbf{0}}^{k}+\Delta x \sum_{m=1}^{s} \tilde{b}_{m}^{(1)} \partial_{x} U_{m k}, \\
w_{\mathbf{1}}^{k}=w_{\mathbf{0}}^{k}+\Delta x \sum_{m=1}^{s} \tilde{b}_{m}^{(2)} \partial_{x} W_{m k} \\
u_{m}^{\mathbf{1}}=u_{m}^{\mathbf{0}}+\Delta t \sum_{k=1}^{r} b_{k}^{(1)} \partial_{t} U_{m k}, \\
v_{m}^{\mathbf{1}}=v_{m}^{\mathbf{0}}+\Delta t \sum_{k=1}^{r} b_{k}^{(2)} \partial_{t} V_{m k}, \\
\partial_{t} U_{m k}=V_{m k}, \partial_{x} U_{m k}=W_{m k}, \\
\partial_{t} V_{m k}=\partial_{x} W_{m k}-G^{\prime}\left(U_{m k}\right),
\end{gathered}
$$

under the assumption that

$$
\begin{aligned}
& \sum_{j=1}^{r} a_{k j}^{(1)}=\sum_{j=1}^{r} a_{k j}^{(2)}=d_{k} \\
& \sum_{n=1}^{s} \tilde{a}_{m n}^{(1)}=\sum_{n=1}^{s} \tilde{a}_{m n}^{(2)}=c_{m} .
\end{aligned}
$$


Here the notation in the following sense is

$$
\begin{gathered}
U_{m k} \approx u\left(c_{m} \Delta x, d_{k} \Delta t\right), \\
\partial_{t} U_{m k} \approx \partial_{t} u\left(c_{m} \Delta x, d_{k} \Delta t\right), \quad \partial_{x} U_{m k} \approx \partial_{x} u\left(c_{m} \Delta x, d_{k} \Delta t\right), \\
u_{\mathbf{0}}^{k} \approx u\left(0, d_{k} \Delta t\right), \quad u_{\mathbf{1}}^{k} \approx u\left(\Delta x, d_{k} \Delta t\right), \\
u_{m}^{\mathbf{0}} \approx u\left(c_{m} \Delta x, 0\right), \quad u_{m}^{\mathbf{1}} \approx u\left(c_{m} \Delta x, \Delta t\right) .
\end{gathered}
$$

Theorem 3.2. In the method (3.4)-(3.13), assume that (3.14), (3.15) and

$$
\begin{aligned}
& b_{k}^{(1)}=b_{k}^{(2)}=b_{k}, \tilde{b}_{m}^{(1)}=\tilde{b}_{m}^{(2)}=\tilde{b}_{m}, \\
& \tilde{b}_{m}^{(1)} \tilde{b}_{n}^{(2)}-\tilde{b}_{m}^{(1)} \tilde{a}_{m n}^{(2)}-\tilde{b}_{n}^{(2)} \tilde{a}_{n m}^{(1)}=0, \\
& b_{k}^{(1)} b_{j}^{(2)}-b_{k}^{(1)} a_{k j}^{(2)}-b_{j}^{(2)} a_{j k}^{(1)}=0
\end{aligned}
$$

hold for $m, n=1,2, \ldots, s, k, j=1,2, \ldots, s$. Then the method (3.4) (3.13) is multisymplectic with a discrete multi-symplectic conservation law

$$
\Delta t \sum_{k=1}^{r} b_{k}\left(d u_{\mathbf{1}}^{k} \wedge d w_{\mathbf{1}}^{k}-d u_{\mathbf{0}}^{k} \wedge d w_{\mathbf{0}}^{k}\right)=\Delta x \sum_{m=1}^{s} \tilde{b}_{m}\left(d u_{m}^{\mathbf{1}} \wedge d v_{m}^{\mathbf{1}}-d u_{m}^{\mathbf{0}} \wedge d w_{m}^{\mathbf{0}}\right) .
$$

Proof. It follows from (3.4)-(3.13) and the conditions (3.14)-(3.18) that

$$
\begin{aligned}
& d u_{\mathbf{1}}^{k} \wedge d w_{\mathbf{1}}^{k}-d u_{\mathbf{0}}^{k} \wedge d w_{\mathbf{0}}^{k} \\
& =\Delta x \sum_{m=1}^{s} \tilde{b}_{m}\left(d U_{m k} \wedge d\left(\partial_{x} W_{m k}\right)\right)
\end{aligned}
$$

and

$$
\begin{aligned}
& d u_{m}^{\mathbf{1}} \wedge d v_{m}^{\mathbf{1}}-d u_{m}^{\mathbf{0}} \wedge d v_{m}^{\mathbf{0}} \\
& =\Delta t \sum_{k=1}^{r} b_{k}\left(d U_{m k} \wedge d\left(\partial_{t} V_{m k}\right)\right) .
\end{aligned}
$$

On the other hand, (3.13) implies that

$$
d U_{m k} \wedge d\left(\partial_{x} W_{m k}\right)=d U_{m k} \wedge d\left(\partial_{t} V_{m k}\right) .
$$

From (3.20), (3.21), and (3.22), the discrete conservation law (3.19) is proved. This completes the proof.

Remark 3.3. (3.12) and (3.13) imply that (3.14) and (3.15), in essence, are not necessary for the characterization (3.16) - (3.18) of multi-symplectic partitioned RungeKutta methods (3.4)-(3.13).

\section{The CONSERVATION OF ENERGY AND MOMENTUM}

It has been shown, by S. Reich in 9] (also see [2]), that multi-symplectic GaussLegendre schemes preserve both the discrete energy and momentum conservation laws exactly for linear Hamiltonian PDEs. In this section we show that the scheme (1.7) - (1.11) preserves the discrete energy and momentum conservation laws exactly for linear Hamiltonian PDEs

$$
M z_{t}+K z_{x}=\nabla_{z} S(z)
$$


where $M$ and $K$ are skew-symmetric matrices, $S(z)=\frac{1}{2} z^{T} A z$, and $A$ is a symmetric matrix. The equation (4.1) has the energy conservation law

$$
\begin{aligned}
\partial_{t} E(z)+\partial_{x} F(z) & =0, \\
\partial_{t} I(z)+\partial_{x} G(z) & =0,
\end{aligned}
$$

where

$$
\begin{aligned}
E(z) & =\frac{1}{2} z^{T} A z-\frac{1}{2} \partial_{x} z^{T} K^{T} z \\
F(z) & =\frac{1}{2} \partial_{t} z^{T} K^{T} z \\
G(z) & =\frac{1}{2} z^{T} A z-\frac{1}{2} \partial_{t} z^{T} M^{T} z \\
I(z) & =\frac{1}{2} \partial_{x} z^{T} M^{T} z .
\end{aligned}
$$

Theorem 4.1. Under the assumptions of Theorem 1.2, if the matrices of RK methods in the method (1.7)-(1.11) are invertible, then the method (1.7)-(1.11) has a discrete energy conservation law

$$
\Delta x \sum_{m=1}^{s} \tilde{b}_{m}\left(E\left(z_{m}^{\mathbf{1}}\right)-E\left(z_{m}^{\mathbf{0}}\right)\right)+\Delta t \sum_{k=1}^{r} b_{k}\left(F\left(z_{\mathbf{1}}^{k}\right)-F\left(z_{\mathbf{0}}^{k}\right)\right)=0
$$

and a discrete momentum conservation law

$$
\Delta x \sum_{m=1}^{s} \tilde{b}_{m}\left(I\left(z_{m}^{\mathbf{1}}\right)-I\left(z_{m}^{\mathbf{0}}\right)\right)+\Delta t \sum_{k=1}^{r} b_{k}\left(G\left(z_{\mathbf{1}}^{k}\right)-G\left(z_{\mathbf{0}}^{k}\right)\right)=0 .
$$

Proof. First of all, we introduce the system

$$
\begin{aligned}
& \partial_{x} Z_{m k}=\left(\partial_{x} z\right)_{m}^{\mathbf{0}}+\Delta t \sum_{j=1}^{r} a_{k j} \partial_{t}\left(\partial_{x} Z_{m j}\right), \\
& \left(\partial_{x} z\right)_{m}^{\mathbf{1}}=\left(\partial_{x} z\right)_{m}^{\mathbf{0}}+\Delta t \sum_{k=1}^{r} b_{k} \partial_{t}\left(\partial_{x} Z_{m k}\right), \\
& \partial_{t} Z_{m k}=\left(\partial_{t} z\right)_{\mathbf{0}}^{k}+\Delta x \sum_{n=1}^{s} \tilde{a}_{m n} \partial_{x}\left(\partial_{t} Z_{n k}\right), \\
& \left(\partial_{x} z\right)_{\mathbf{1}}^{k}=\left(\partial_{x} z\right)_{\mathbf{0}}^{k}+\Delta x \sum_{m=1}^{r} \tilde{b}_{m} \partial_{x}\left(\partial_{t} Z_{m k}\right),
\end{aligned}
$$

where $\left(\partial_{x} z\right)_{m}^{\mathbf{0}}$ and $\left(\partial_{t} z\right)_{\mathbf{0}}^{k}$ satisfy

$$
\begin{aligned}
& z_{m}^{\mathbf{0}}=z_{0}^{\mathbf{0}}+\Delta x \sum_{n=1}^{s} \tilde{a}_{m n}\left(\partial_{x} z\right)_{n}^{\mathbf{0}} \\
& z_{\mathbf{0}}^{k}=z_{\mathbf{0}}^{k}+\Delta t \sum_{j=1}^{r} a_{k j}\left(\partial_{t} z\right)_{\mathbf{0}}^{k}
\end{aligned}
$$

respectively, and

$$
\begin{aligned}
& \partial_{t}\left(\partial_{x} Z_{m k}\right) \approx \partial_{t x} z\left(c_{m} \Delta x, d_{k} \Delta t\right), \\
& \partial_{x}\left(\partial_{t} Z_{m k}\right) \approx \partial_{x t} z\left(c_{m} \Delta x, d_{k} \Delta t\right) .
\end{aligned}
$$


Because matrices $A=\left(a_{k j}\right)_{r \times r}$ and $\tilde{A}=\left(\tilde{a}_{m n}\right)_{s \times s}$ are invertible, we have

$$
\partial_{t}\left(\partial_{x} Z_{m k}\right)=\partial_{x}\left(\partial_{t} Z_{m k}\right) \text {. }
$$

In fact, (1.9), (4.6), and (4.10) imply that

$$
Z_{m k}=z_{\mathbf{0}}^{k}+z_{m}^{\mathbf{0}}-z_{0}^{\mathbf{0}}+\Delta x \Delta t \sum_{j=1}^{r} \sum_{n=1}^{s} a_{k j} \tilde{a}_{m n} \partial_{t}\left(\partial_{x} Z_{n j}\right) .
$$

Similarly, (1.7), (4.8), and (4.11) imply that

$$
Z_{m k}=z_{m}^{\mathbf{0}}+z_{\mathbf{0}}^{k}-z_{0}^{\mathbf{0}}+\Delta x \Delta t \sum_{j=1}^{r} \sum_{n=1}^{s} a_{k j} \tilde{a}_{m n} \partial_{x}\left(\partial_{t} Z_{n j}\right) .
$$

From (4.13) and (4.14), we conclude that (4.12) holds for $m=1,2, \ldots, s$ and $k=1,2, \ldots, r$.

From the assumptions, we have

$$
\begin{aligned}
& \frac{1}{2}\left(z_{\mathbf{1}}^{k}\right)^{T} K^{T}\left(\partial_{t} z\right)_{\mathbf{1}}^{k}=\frac{1}{2}\left(z_{\mathbf{0}}^{k}\right)^{T} K^{T}\left(\partial_{t} z\right)_{\mathbf{0}}^{k} \\
& +\frac{\Delta x}{2} \sum_{m=1}^{s} \tilde{b}_{m}\left(Z_{m k}\right)^{T} K^{T} \partial_{x}\left(\partial_{t} Z_{m k}\right)+\frac{\Delta x}{2} \sum_{m=1}^{s} \tilde{b}_{m} \partial_{x}\left(Z_{m k}\right)^{T} K^{T}\left(\partial_{t} Z_{m k}\right) .
\end{aligned}
$$

Therefore,

$$
\begin{aligned}
\frac{F\left(z_{\mathbf{1}}^{k}\right)-F\left(z_{\mathbf{0}}^{k}\right)}{\Delta x}= & \frac{1}{2} \sum_{m=1}^{s} \tilde{b}_{m}\left(Z_{m k}\right)^{T} K^{T} \partial_{x}\left(\partial_{t} Z_{m k}\right) \\
& +\frac{1}{2} \sum_{m=1}^{s} \tilde{b}_{m} \partial_{x}\left(Z_{m k}\right)^{T} K^{T}\left(\partial_{t} Z_{m k}\right) .
\end{aligned}
$$

A similar (but a little bit tedious) calculation leads to

$$
\begin{aligned}
\frac{E\left(z_{m}^{\mathbf{1}}\right)-E\left(z_{m}^{\mathbf{0}}\right)}{\Delta t}= & -\frac{1}{2} \sum_{k=1}^{r} b_{k}\left(Z_{m k}\right)^{T} K^{T} \partial_{t}\left(\partial_{x} Z_{m k}\right) \\
& -\frac{1}{2} \sum_{k=1}^{r} b_{k} \partial_{x}\left(Z_{m k}\right)^{T} K^{T}\left(\partial_{t} Z_{m k}\right) .
\end{aligned}
$$

This means that (4.4) holds. Analogously, we show that (4.5) holds. The proof is finished.

Remark 4.2. The discrete conservation of energy and momentum for (2.4)-(2.12) can be discussed in a similar way, but with tedious calculations.

\section{CONClusion}

Theorem 1.2 tells us that concatenating two symplectic Runge-Kutta methods probably produces a multi-symplectic integrator with the order that we need. Theorem 2.1 provides theoretically many more ways of constructing multi-symplectic integrators by using partitioned Runge-Kutta methods. For example, a multisymplectic integrator of the wave equation can be produced by using the Lobatto IIIA-IIIB pair to discretize the equation both in time and in space directions. 


\section{REFERENCES}

1. J.C.Butcher, The Numerical Analysis of Ordinary Differential Equations. Runge-Kutta and General Linear Methods, John Wiley \& Sons, Chichester, 1987. MR0878564 (88d:65002)

2. Th.J.Bridges and S.Reich, Multi-symplectic integrator: numerical schemes for Hamiltonian PDEs that conserve symplecticity, Physics Letters A, 284(4-5) (2001), 184-193. MR.1854689 (2002g:65166)

3. G.J.Cooper, Stability of Runge-Kutta methods for trajectory problems, IMA J. Numer. Anal., 7 (1987), 1-13. MR0967831 (90d:65133)

4. D.B.Duncan, Symplectic finite difference approximations of the nonlinear Klein-Gordon equation, SIAM J. Numer. Anal., 34 (1997), 1742-1760. MR1472194(98m:65139)

5. J.de Frutos \& J.M.Sanz-Serna, Split-step spectral schemes for nonlinear Dirac systems, J. Comput. Phys., 83 (1989), 407-423. MR:1013060 (91f:81021)

6. P.Görtz and R.Scherer, Hamiltonian systems and symplectic integrators, Nonlinear Analysis TMA, 30 (1997), 1887-1892. MR.1490098 (98j:65049)

7. A.Iserles, A First Course in the Numerical Analysis of Differential Equations, Cambridge University Press, Cambridge, 1996. MR1384977 (97m:65003)

8. J.E.Marsden \& M.West, Discrete mechanics and variational integrators, Acta Numerica, 10 (2001), 1-158. MR2009697 (2004h:37130)

9. S.Reich, Multi-symplectic Runge-Kutta collocation methods for Hamiltonian wave equations, J. Comput. Phys., 157 (2000) 2, 473-499. MR1739109 (2001a:65087)

10. J.M.Sanz-Serna, Runge-Kutta schemes for Hamiltonian systems, BIT, 28 (1988), 877-883. MR0972812 (90b:65145)

11. J.M.Sanz-Serna, The numerical integration of Hamiltonian systems. In: Computational Ordinary Differential Equations, ed. by J.R. Cash \& I.Gladwell, Clarendon Press, Oxford, (1992) 437-449. MR 1387155

12. J.M.Sanz-Serna and M.P.Calva, Numerical Hamiltonian Systems, Chapman \& Hall, London, 1994. MR.1270017 (95f:65006)

13. G.Sun, A simple way of constructing symplectic Runge-Kutta methods, J. Comput. Math., 18 (2000), 61-68. MR1741173 (2000j:37118)

14. G.Sun, Symplectic partitioned Runge-Kutta methods, J. Comput. Math., 11 (1993), 365-372. MR.1252773 (95a:65113)

15. Y.B.Suris, On the conservation of the symplectic structure in the numerical solution of Hamiltonian systems (in Russian), In: Numerical Solution of Ordinary Differential Equations, ed. S.S. Filippov, Keldysh Institute of Applied Mathematics, USSR Academy of Sciences, Moscow, 1988, 148-160.

16. Y.B.Suris, Hamiltonian methods of Runge-Kutta type and their variational interpolation (in Russian), Math. Model., 2 (1990), 78-87. MR1064467 (92b:65051)

State Key Laboratory of Scientific and Engineering Computing, Institute of Computational Mathematics and Scientific/Engineering Computing, Academy of Mathematics and System Sciences, Chinese Academy of Sciences, P.O.Box 2719, Beijing 100080, PeoPLE'S Republic OF CHINA

E-mail address: hjl@lsec.cc.ac.cn

Institute of Mathematics, Academy of Mathematics and System Sciences, Chinese Academy of Sciences, Beijing 100080, People's Republic of China

Current address: Department of Mathematics, The Chinese University of Hong Kong, Hong Kong, People's Republic of China

E-mail address: hyliu@math.cuhk.edu.hk

Institute of Mathematics, Academy of Mathematics and System Sciences, Chinese Academy of Sciences, Beijing 100080, People's Republic of China

E-mail address: sung@mail.amss.ac.cn 